\title{
Enantioselective total synthesis of the novel tricyclic sesquiterpene (-)-sulcatine G. Absolute configuration of the natural product
}

\author{
Goverdhan Mehta* and K. Sreenivas \\ Department of Organic Chemistry, Indian Institute of Science, Bangalore 560 012, India
}

\begin{abstract}
An enantioselective total synthesis of (-)-sulcatine G 4 from the readily available (+)-diquinane diol $\mathbf{6}$ has been accomplished. This leads to the establishment of the absolute configuration of the natural product (+)-sulcatine $\mathrm{G}$ as $\mathbf{1}$.
\end{abstract}

During the past decade, novel terpene skeleta embodying the 4-5-5 ring fused tricarbocyclic core have been encountered in Nature from diverse sources. Among the very few known examples of terpenoid natural products bearing this ring system are sulcatine $G \mathbf{1}$ from a Basidiomycetes fungi, ${ }^{1}$ kelsoene $\mathbf{2}$ from a tropical marine sponge Cymbastela hooperi, ${ }^{2 \mathrm{a}}$ liverworts Ptychanthus striatus, ${ }^{2 \mathrm{~b}, \mathrm{c}}$ Calypogeia muelleriana ${ }^{2 \mathrm{~d}}$ and Tritomaria quinquedentata ${ }^{2 \mathrm{e}}$ and poduran 3 from the springtail Podura aquatica. ${ }^{3}$ The structural novelty and interesting biosynthetic origin of these natural products have aroused considerable synthetic interest in the past few years. ${ }^{4,5}$ We too have been enticed by these natural products and have accomplished the total synthesis of racemic, (+)- and (-)-kelsoene 2 . $^{4 \mathrm{a}-\mathrm{c}}$ Continuing our efforts in the area, a total synthesis of racemic sulcatine G 1 has been reported recently by us, ${ }^{5}$ fully securing its formulation which had been earlier deduced ${ }^{1}$ mainly from the analysis of the NMR data. However, the absolute configuration of sulcatine $G$ remains unknown as the functionality profile of the natural product does not permit ready recourse to chiro-optical methods of absolute configuration determination. Herein, we wish to describe an enantioselective synthesis of (-)-sulcatine G 4, which establishes the absolute configuration of the naturally occurring (+)- sulcatine $\mathrm{G}$ as $\mathbf{1}$.

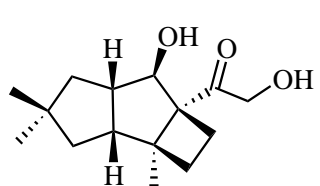

(+)-Sulcatine $\mathbf{G} 1$

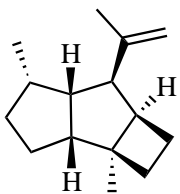

(+)-Kelsoene 2
We have recently shown that endo,endo-cis-bicyclo[3.3.0]octane-2,6-diol rac-6, obtained in two steps from commercially available 1,5-cyclooctadiene 5 via $\mathrm{Pd}^{2+}$-mediated transannular diacetoxylation and hydrolysis, ${ }^{6}$ on lipase-catalyzed enantiomer selective transesterification in an organic medium furnished diol $(+)-6 \quad(>98 \%$ ee) and diacetate $(+)-7 \quad(>99 \%$ ee) in preparatively useful yields (Scheme 1$).{ }^{4 \mathrm{c}}$ For our projected enantioselective synthesis of sulcatine $G$, diol (+)-6 was employed and elaborated to diquinane ketone (-)-9, through the intermediacy of $(-)-\mathbf{8}$, as described by us recently ${ }^{5}$ (Scheme 2). ${ }^{7}$ This is the first enantioselective preparation of $(-)-9$, which in racemic form has been previously employed in the synthesis of triquinane natural products ${ }^{8}$ and may find further applications in chiral synthesis of terpenoid natural products.

Diquinane (-)-9 was further elaborated to the $\alpha$-carbomethoxycyclopentenone (-)-10 as shown in Scheme $3 .^{7}$ Further evolution of (-)-10 to the tricyclic bridgehead vinyl compound (-)-11, involved [2+2]-photocycloaddition as a pivotal step to append the cyclobutane ring and generate the desired 4-5-5 fused tricyclic framework. With tricyclic (-)-11 having all the 15 -carbons of the natural product in hand, the remaining task was to harness the vinyl group to access the oxy-func-

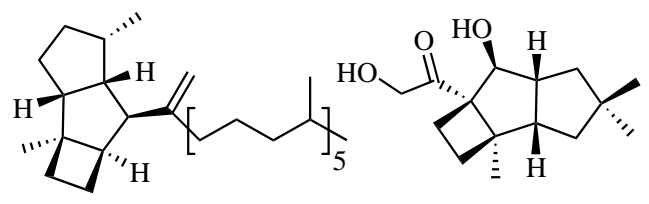

Poduran 3

\footnotetext{
* Corresponding author.
} 


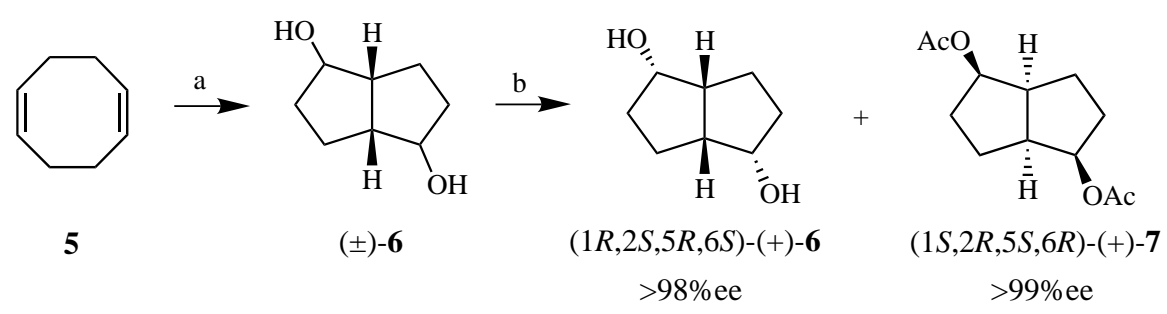

Scheme 1. Reagents and conditions: (a) i. $\mathrm{PdCl}_{2}, \mathrm{~Pb}(\mathrm{OAc})_{4}, \mathrm{AcOH}, \mathrm{rt}, 72 \mathrm{~h}, 70 \%$; ii. $\mathrm{KOH}, \mathrm{MeOH}, \mathrm{rt}, 3$ h, $95 \%$; (b) vinyl acetate, Amano lipase PS-30, ${ }^{t}$ BuOMe, rt, 6 days, $82 \%$.<smiles>O[C@H]1CC[C@H]2[C@@H](O)CC[C@H]12</smiles>

$(+)-6$

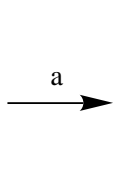

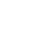<smiles>O=C1CC[C@H]2[C@@H](OCc3ccccc3)CC[C@H]12</smiles>$$
\text { ( }
$$<smiles>CC(C)[C@H]1CC[C@H]2[C@@H](O)CC(C)(C)C(=O)[C@@H]2C1</smiles>

$(-)-8$

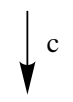<smiles>CC(C)[C@]1(C)C[C@H]2CCC(=O)[C@H]2C[C@H]1C</smiles>

$(-)-9$

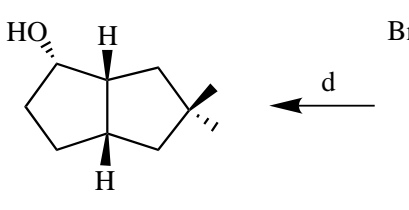<smiles>CC1(C)C[C@H]2[C@@H](OBr)CC[C@H]2C12SCCS2</smiles>

Scheme 2. Reagents and conditions: (a) i. $\mathrm{NaH}, \mathrm{BnBr}, \mathrm{Bu}_{4} \mathrm{~N}^{+} \mathrm{I}^{-}$, THF, rt, $12 \mathrm{~h}, 87 \%$; ii. PCC, DCM, rt, $94 \%$; (b) $\mathrm{KO}{ }^{t} \mathrm{Bu},{ }^{t} \mathrm{BuOH}$, MeI, $0^{\circ} \mathrm{C}-\mathrm{rt}, 8$ h, 91\%; (c) i. $\left(\mathrm{CH}_{2} \mathrm{SH}\right)_{2}$, PTSA, benzene, 96\%; (d) Raney-Ni, EtOH, reflux, 6 h, 92\%; (e) PCC, DCM, rt, 3 h, $90 \%$.

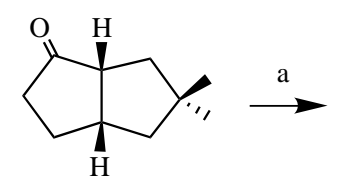

(-)-9<smiles>CCCC[C@@]1(C)C[C@@H]2C=C(C(=O)OC)C(=O)C(=O)[C@@H]2C1</smiles><smiles>CC(=O)C1=C(C)[C@H]2CC(C)(C)C[C@H]2C1=O</smiles>

(-)-10

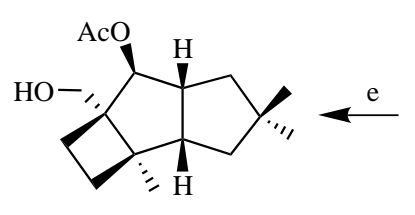<smiles>CC1(C)C[C@H]2[C@@H](C1)[C@@H](O)[C@]1(CO)CC[C@]21C</smiles><smiles>CC(=O)[C@]12C(=O)[C@H]3CC(C)(C)C[C@H]3[C@H]1[C@H](Cl)[C@H]2Cl</smiles><smiles>CC(C)CC(C)C</smiles>

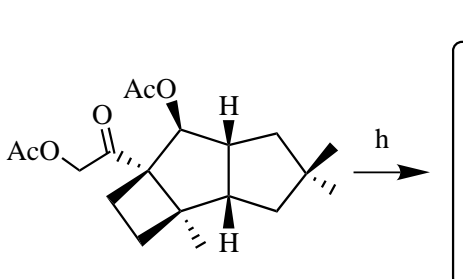

(-)-12

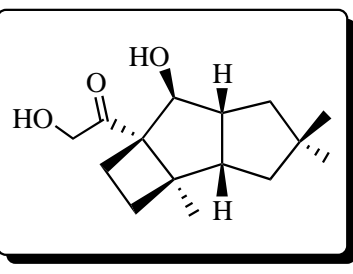

(-)-Sulcatine G 4

Scheme 3. Reagents and conditions: (a) i. $\mathrm{NaH},(\mathrm{MeO})_{2} \mathrm{CO}$, benzene, reflux, $5 \mathrm{~h}, 85 \%$; ii. $\mathrm{NaH}, \mathrm{PhSeCl}, \mathrm{THF}, 0^{\circ} \mathrm{C}, 15 \mathrm{~min} ;$ iii. $30 \%$ $\mathrm{H}_{2} \mathrm{O}_{2}, \mathrm{DCM}, 0^{\circ} \mathrm{C}, 15 \mathrm{~min}, 82 \%$ (two steps); (b) i. MeMgI, CuI, Et ${ }_{2} \mathrm{O},-10^{\circ} \mathrm{C}, 2 \mathrm{~h}, 96 \%$; ii. $\mathrm{NaH}, \mathrm{PhSeCl}, \mathrm{THF}, 0^{\circ} \mathrm{C}, 15 \mathrm{~min}$; iii. $30 \% \mathrm{H}_{2} \mathrm{O}_{2}, \mathrm{DCM}, 0^{\circ} \mathrm{C}, 15 \mathrm{~min}, 50 \%$, (two steps); (c) trans-1,2-dichloroethylene, $\mathrm{C}_{6} \mathrm{H}_{12}, h v$, Pyrex, rt, 6 h, 95\%; (d) i. DIBAL-H, DCM, rt, 3 h, ii. sodium naphthalenide, DME, rt, 1 h; iii. $\mathrm{H}_{2}, \mathrm{PtO}_{2}$, EtOAc, $1 \mathrm{~h}, 60 \%$ (three steps); (e) i. ${ }^{t} \mathrm{BDMS}-\mathrm{Cl}$, imidazole, DMAP, DCM, 8 h, rt, 92\%; ii. Ac 2 O, DMAP, DCM, rt, 20 h, 100\%; iii. $2 \mathrm{~N} \mathrm{H}_{2} \mathrm{SO}_{4}, \mathrm{MeOH}-\mathrm{H}_{2} \mathrm{O}(4: 1)$, rt, 2 h, $90 \%$; (f) i. PCC, DCM, rt, 2 h, $91 \%$; ii. $\mathrm{MePPh}_{3} \mathrm{I}, \mathrm{KO}^{t} \mathrm{Bu}, \mathrm{THF}, 0^{\circ} \mathrm{C}, 10 \mathrm{~min}, 92 \%$; (g) i. OsO $\mathrm{O}_{4}, \mathrm{NMMO} \mathrm{Me}_{2} \mathrm{CO}-\mathrm{H}_{2} \mathrm{O}(4: 1)$, rt, 2 h, $86 \%$; ii. Ac $2 \mathrm{O}$, DMAP, DCM, $0^{\circ} \mathrm{C}, 30 \mathrm{~min}, 100 \%$; iii. PCC, DCM, rt, $6 \mathrm{~h}, 77 \%$; (h) i. $\mathrm{Sc}(\mathrm{OTf})_{3}, \mathrm{MeOH}-\mathrm{H}_{2} \mathrm{O}(4: 1), 47^{\circ} \mathrm{C}, 8 \mathrm{~h}$; ii. $8 \%$ $\mathrm{KOH}-\mathrm{MeOH},-10^{\circ} \mathrm{C}, 3 \mathrm{~h}, 61 \%$ (two steps). 
tionalization present in the natural product and sulcatine $\mathrm{G}$ diacetate (-)-12 was readily realized, Scheme $3 .^{7}$ Finally, acetate hydrolysis as reported previously ${ }^{5}$ furnished (-)-sulcatine $\mathrm{G} \mathrm{4},[\alpha]_{\mathrm{D}}-40\left(c 0.25, \mathrm{CHCl}_{3}\right)$, which was spectroscopically identical with the natural product but had opposite specific rotation to that reported for the naturally occurring sulcatine $\mathrm{G} 1[\alpha]_{\mathrm{D}}+44.5(c) 0.15$, $\left.\mathrm{CHCl}_{3}\right){ }^{1}$ This established the absolute configuration of the natural product as $\mathbf{1}$.

In summary, we have outlined a stereo- and enantiocontrolled synthesis of the sesquiterpene (-)-sulcatine $\mathrm{G} \mathbf{4}$ from a readily available chiral diquinane diol (+)-6, which unambiguously establishes the absolute configuration of the natural product as depicted in $\mathbf{1}$. Since, sulcatine G (+)-1 is biogenetically related to illudins and related sesquiterpenoids which are also found in Basidiomycetes fungi, determination of the absolute configuration of $\mathbf{1}$ has a bearing on the absolute configuration of other members of this group.

\section{Acknowledgements}

We thank JNCASR for the financial support. One of us (K.S.) thanks UGC for the award of a research fellowship.

\section{References}

1. Arnone, A.; Nasini, G.; Vajna de Pava, O. J. Chem.
Soc., Perkin Trans. 1 1993, 2723.

2. (a) Konig, G.; Wright, A. D. J. Org. Chem. 1997, 62, 3837; (b) Nabeta, K.; Yamamoto, K.; Hashimoto, M.; Koshino, H.; Funatsuki, K.; Katoh, K. Chem. Commun. 1998, 1485; (c) Hashimoto, T.; Ikeda, H.; Takaoka, S.; Tanaka, M.; Asakawa, Y. Phytochemistry 1999, 52, 501; (d) Warmers, U.; Wihstutz, K.; Bulow, N.; Fricke, C.; Konig, W. A. Phytochemistry 1998, 49, 1723; (e) Warmers, U.; Konig, W. A. Phytochemistry 1999, 52, 1519.

3. Schulz, S.; Messer, C.; Dettner, K. Tetrahedron Lett. 1997, 38, 2077.

4. (a) Mehta, G.; Sreenivas, K. Tetrahedron Lett. 1999, 40, 4877; (b) Mehta, G.; Sreenivas, K. Synlett 1999, 555; (c) Mehta, G.; Sreenivas, K. Tetrahedron Lett. 2001, 42, 2855; (d) Piers, E.; Orellana, A. Synthesis 2001, 2138; (e) Fietz-Razavian, S.; Schulz, S.; Dix, I.; Jones, P. G. Chem. Commun. 2001, 2154.

5. Mehta, G.; Sreenivas, K. Chem. Commun. 2001, 1892.

6. (a) Henry, P. M.; Davies, M.; Ferguson, G.; Philips, S.; Restivo, R. Chem. Commun. 1974, 112; (b) Mehta, G.; Rao, K. V. Ind. J. Chem. 1991, 30B, 457.

7. All new compounds reported here were duly characterized on the basis of spectroscopic (IR, ${ }^{1} \mathrm{H}$ and ${ }^{13} \mathrm{C}$ NMR) and analytical data and their specific rotations were determined.

8. Cossy, J.; Belotti, D.; Pete, J. P. Tetrahedron Lett. 1987, 28 , 4547. For a recent review on the synthesis of polyquinane natural products, see: Mehta, G.; Srikrishna, A. Chem. Rev. 1997, 97, 671. 